\title{
Uma Ontologia de Apoio ao Ensino de Experimentação em Engenharia de Software
}

\author{
Carlos Danilo Luz ${ }^{1}$ (mestrando), Edson OliveiraJr ${ }^{1}$ (orientador) \\ Igor F. Steinmacher ${ }^{2}$ (coorientador) \\ ${ }^{1}$ Mestrado Acadêmico em Ciência da Computação \\ Programa de Pós-Graduação em Ciência da Computação
}

Departamento de Informática - Universidade Estadual de Maringá (UEM)

Avenida Colombo, 5790 - CEP 87020-900 - Maringá - PR - Brasil

Ingresso: 03/2018 - Qualificação: 09/2019 - Previsão Defesa: 03/2021

carlos.danilo.luz@gmail.com, edson@din.uem.br

${ }^{2}$ Northern Arizona University (NAU)

igor.steinmacher@nau.edu

\begin{abstract}
Resumo. O uso de experimentação para avaliação empírica das atividades de desenvolvimento de software é uma prática comum nos dias de hoje. Tanto para a indústria de software como em pesquisas acadêmicas, a experimentação se mostra fundamental para a compreensão do ciclo de vida de um software, considerando um conjunto de atividades sistemáticas apoiadas por técnicas estatísticas. Por mais que a comunidade científica de Engenharia de Software (ES) venha utilizando experimentação para a avaliação de teorias e tecnologias, ainda há carência de metodologias e materiais de ensino específicos para experimentação em ES (ESE), o que, consequentemente, ameaça o planejamento, a condução e a disseminação adequada de experimentos e suas futuras reproduções. Assim, esta pesquisa tem como objetivo especificar uma ontologia de apoio ao ensino de experimentação em ES, considerando o desenvolvimento, uso e reúso de Recursos Educacionais Abertos (REA). Tal ontologia será capaz de formalizar os conceitos de ensino de ESE e REAs, além de contribuir com a comunidade de ES na produção e disseminação de materiais de ensino e aprendizagem.
\end{abstract}

\section{Eventos CBSoft: SBES e SBCARS}

\section{Caracterização do Problema}

Experimentação é a base do método científico, uma forma sistemática de explorar o mundo [Forsyth 2018]. Embora os experimentos científicos sejam normalmente associados a laboratórios, eles podem ocorrer em todo o lugar, a qualquer tempo, inclusive no desenvolvimento de software [Forsyth 2018].

Em Ciência da Computação, a Experimentação em Engenharia de Software (ESE) desempenha um papel central nas comunidades de pesquisadores e profissionais de engenharia de software (ES). A ESE destina-se a promover evidência de uma certa teoria ou tecnologia relacionada ao ciclo de vida de desenvolvimento de software [Wohlin et al. 2012]. 
É fundamental evidenciar como o conhecimento adquirido com um experimento pode ser importante no contexto de desenvolvimento mais amplo. Experimentos controlados podem ser importantes também sobre a perspectiva de pesquisa ou de uma perspectiva de transferência de tecnologia. De qualquer forma, há uma necessidade de melhorar a forma como ensinamos e conduzimos estudos em engenharia de software [Wohlin 2007].

De acordo com [Kuhrmann 2017] a engenharia de software empírica pode ser aplicada a para: (i) analisar/compreender fenômenos no desenvolvimento de software, (ii) identificar/avaliar os pontos fortes e fracos das abordagens de engenharia de software, e (iii) investigar o estado da arte / prática para identificar soluções/abordagens promissoras.

Um dos fatores que acredita-se contribuir para isso é o fato de haver pouca discussão sobre o ensino de ESE na literatura e de metodologias que guiem tal ensino. Assim, uma das formas de contribuir para o ensino de ESE é a formalização dos conceitos de experimentação por meio de ontologias e a sua integração com conceitos de desenvolvimento, uso e reúso de Recursos Educacionais Abertos (REA).

\section{Fundamentação Teórica}

\subsection{Ensino de Experimentação em Engenharia de Software}

Segundo as diretrizes do MEC [MEC 2015, p.10] "O ensino de Engenharia de Software em cursos de graduação pode dar origem a várias disciplinas com diferentes ênfases. A origem dessas disciplinas pode ter como motivação diferentes classificações didáticas: aspectos gerenciais, aspectos técnicos, aspectos teóricos e aspectos experimentais".

$\mathrm{Na}$ literatura internacional são registradas diversas discussões sobre problemas no processo ensino aprendizagem na área de ES, podendo claramente ser observada a necessidade de maior integração e cooperação no ensino-aprendizagem de disciplinas da área de ES, conforme apresentado por [Paiva 2011]. Além disso [Host 2002] afirma que, como em muitos outros campos de pesquisa aplicada, há uma conexão entre educação e métodos empíricos em ES. Em muitos casos, os estudantes de ES estão envolvidos em estudos empíricos, esta prática é válida ao analisarmos que através do envolvimento dos alunos em tais estudos podemos proporcionar aos mesmos a práxis pedagógica em relacionar os conteúdos teóricos apresentado no decorrer das disciplinas com as práticas vivenciadas no mundo real, como também desenvolver habilidade e competências sobre pesquisa acadêmica e análise de dados.

Segundo [Wohlin 2007] existem diferentes maneiras de ensinar ESE em um determinado currículo, das quais destacam-se as duas seguintes: integrada a cursos de ES, nos quais ESE é parte de outras disciplinas e é analisada com base em avaliações de aprendizagem e projetos práticos; e como uma disciplina isolada, na qual a maior vantagem está no foco específico em experimentos como trabalhos práticos.

Host [2002] ainda discute a introdução de métodos empíricos sobre o ensino de ES e apresenta exemplos de um experimento controlado para avaliar revisões aos pares. Ademais, Host apresenta diferentes possibilidades de avaliar os conhecimentos dos alunos, por meio de palestras, estudos de literatura, projetos e livros como modo de ensino de ES.

A introdução dos conceitos sobre experimentação em ES nos currículos acadêmicos, espera-se poder formar mão de obra especializada para a indústria com co- 
nhecimentos sobre experimentos. Munezero et al [2017] acreditam que experimentos são realizados a partir de hipóteses a serem testadas em um processo sistemático e bem organizado. Passando, assim, a compreender a formulação da proposta para determinada hipótese, especialmente no desenvolvimento de um novo produto ou serviço.

\subsection{Ontologias}

Uma ontologia é uma especificação de um vocabulário representacional para um domínio compartilhado de discurso - definições de classes, relações, funções e outros objetos. Uma ontologia é mais do que apenas uma coleção de objetos e seus relacionamentos. Isso deve especificar as restrições que existem entre esses objetos e relacionamentos [Siy and Wu 2009].

Ontologias podem ser usadas para permitir que vários aplicativos ou pessoas tenham acesso a fontes heterogêneas de informações que são expressas por meio de vocabulários diversos ou formatos inacessíveis. Uma ontologia de um determinado domínio pode fornecer um vocabulário para especificar os requisitos para um ou mais destinos. $\mathrm{Na}$ verdade, a ontologia é usada como base para a especificação e desenvolvimento de software, permitindo a reutilização do conhecimento [de Almeida Biolchini et al. 2007].

Mesmo havendo uma grande variedade de metodologias para a criação de ontologias, esta tarefa não é trivial do ponto de vista da área de Computação. [Mendonça e Soares [2017] afirmam que "o processo de construção de uma ontologia é complexo, pois envolve a criação de modelos semânticos ou descrições simplificadas da realidade de um dado domínio e também exige dos desenvolvedores conhecimentos técnicos em modelagem conceitual, em lógica formal e em alguns aspectos filosóficos”.

\section{Método de Pesquisa}

O objetivo deste trabalho é o desenvolvimento de uma ontologia para apoio ao ensino de experimentação em ES. Para alcançar tal objetivo proposto nesta pesquisa estão sendo realizadas as etapas apresentadas na Figura 1 e descritas a seguir.

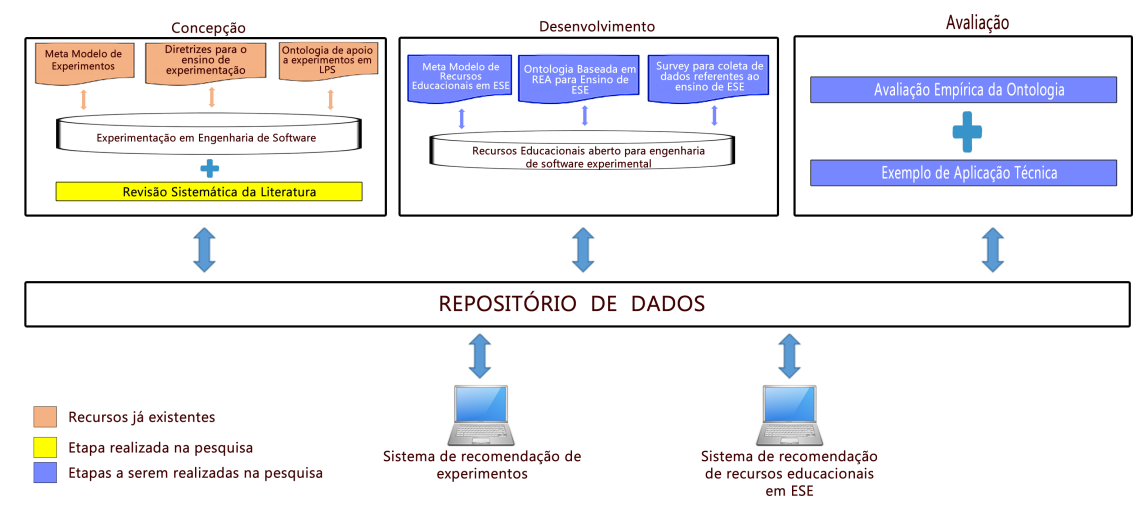

Figura 1. Metodologia de pesquisa adotada 
Concepção: foi realizado um estudo de meta dados de experimentos inicialmente identificados no trabalho de Furtado [2018] e refinados no trabalho de Vignando [2018]. O resultado foi uma ontologia para ESE. Tal ontologia foi clusterizada a partir de um modelo conceitual desenvolvido por Furtado. Além dos trabalhos bases citados para a formulação inicial da concepção do trabalho, o discente realizou uma revisão sistemática da literatura afim de compreender o estado da arte sobre o uso de REA para o ensino de ESE conforme descrito na metodologia, tendo como base de pesquisa os assuntos: sistemas de recomendações de experimentos em ES, ontologias já existentes no domínio da Ciência da Computação e repositórios do domínio a ser trabalhado.

Desenvolvimento: analisou-se a forma como os dados são organizados em um REA, compreendendo os seus princípios, relacionamento e a criação de REAs, por meio de revisão da literatura e pesquisas aplicadas. O desenvolvimento da atual ontologia se diferencia da ontologia específica para formalização dos conceitos de ES experimental, proposta por [Vignando et al. 2020], ao utilizar os conceitos de experimentação em ES como recursos educacionais a serem utilizados por docentes. Ao realizar uma pesquisa sobre as metodologias para construção de ontologias foi possível observar que existem diversas metodologias. Para o presente trabalho foi selecionada a metodologia Methontology a qual foi analisada no processo da RSL e constatado que seus processo previamente definidos seriam adequados para a construção da ontologia pretendida, visto que a mesma apresenta passos bem definidos para o processo de criação de uma ontologia, sendo estes a Especificação, Aquisição de Conhecimento, Conceitualização, Integração, Implementação, Avaliação e Documentação. Como apoio à construção da ontologia, um survey sobre ESE permitirá identificar os principais elementos e conteúdos ministrados no ESE, bem como extrair seus metadados.

Avaliação: ao término do desenvolvimento da ontologia proposta serão realizados estudos qualitativos usando o Modelo de Aceitação de Tecnologia (Technology Acceptance Model - TAM) com a participação de especialistas em experimentação e ensino de ES, afim de compreender se a mesma esta adequada e contém os elementos básicos e necessários para serem utilizados no ensino de ESE.

A ontologia visa contribuir especificamente com a formalização e estruturação de dados e metadados sobre REAs para ESE. Assim, pode-se povoar a ontologia com REAs já existentes (oriundos do survey em execução) e, em trabalhos futuros, um sistema de recomendação poderá fazer inferências em tal ontologia permitindo a recomendação mais precisa de REAs para o ensino de ESE.

\section{Estado Atual do Trabalho}

A partir da realização da revisão sistemática da literatura sobre ensino de experimentação e repositórios de conteúdos acadêmicos para o domínio a ser explorado na pesquisa, notou-se que foram encontrados poucos materiais que poderiam ser utilizados para o ensino de ESE. Foram encontrados artigos relatando sobre o ensino de estudos empíricos em engenharia de software, mas pouco detalhava sobre os processos metodológicos educacionais, assuntos gerais e específicos no ensino de experimentos, conteúdos básicos, material de textual de apoio, como também observou-se a falta de um repositório formal de possíveis materiais para serem utilizados no ensino a discentes, sendo assim, demonstrando uma carência de conteúdos que podem ser utilizados como apoio pedagógico ao 
docente no processo de ensino. Assim, para a fase de elaboração da ontologia neste trabalho, foi conduzido um survey a fim de coletar dados referentes ao ensino de ESE (e.g., referencias básicas, cronograma de estudos, reuso de materiais - materiais próprios ou de terceiros - e tópicos abordados).

O survey segue em fase de coleta de dados 11 Espera-se obter com este survey dados para compor a ontologia e preparar materiais de apoio, como REAs, aos docentes com as devidas formalizações para o ensino ESE.

\section{Contribuições}

Se espera ao final desta pesquisa desenvolver uma ontologia capaz de fornecer subsídios pedagógicos no contexto do ensino de ESE e na preparação de estudantes para o mercado de trabalho. Com isso, formando um capital humano mais adequado e treinado sobre a experimentação de software.

Ainda, tem-se como contribuição a formalização dos conceitos de experimentação e ensino de ESE ao projetar e conduzir experimentos de qualidade, com a finalidade de aproximar estudantes a casos reais da indústria de software. Ainda, pretende-se fomentar a produção e o reúso de materiais de ensino de experimentação por meio da ontologia proposta e dos conceitos de REA.

Espera-se com a pesquisa reunir evidências sobre os processos, métodos e materiais didáticos utilizados pelos docentes no decorrer no ensino de experimentação para que se possa alimentar a ontologia a ser desenvolvida com dados do survey, o que demonstra um potencial interessante para esta pesquisa.

\section{Descrição e Avaliação dos Resultados}

A revisão sistemática da literatura realizada não apresentou resultados sobre a formalização de conteúdos para o ensino de ESE, tal como uma ontologia para a formalização das metodologias para o ensino de experimentação.

Uma das formas da capitação de conhecimento está sendo a realização por intermédio do survey. Atualmente tem-se 25 respostas de docentes que atuam com o ensino de ESE, sendo eles do Brasil, Equador, Alemanha, EUA, Uruguai, Suécia e Holanda.

Ao se analisar os resultados parciais do survey, é possível compreender que em $72 \%$ das respostas obtidas sobre como é ensinado os os conceitos de experimentação em ES são aplicados em disciplinas exclusivas sobre o assunto, 64\% dos materiais produzidos ou utilizados pelos docentes não seguem nenhum tipo de licença aberta, em sua maioria o material de apoio utilizado é o livro de [Wohlin et al. 2012] e 72\% das pessoas que responderam o questionário ensinam experimentação em cursos de Mestrado. Por fim, é compreensível por meio do survey que o método mais utilizado para a avaliação dos decentes é por meio de seminários e projetos práticos.

\section{Comparação com Trabalhos Relacionados}

O artigo de [Alves et al. 2012] consiste na aplicação de experimentos sobre a análise do desenvolvimento de uma estrutura de processo para envolver os alunos como sujeitos

\footnotetext{
1 https://forms.gle/faHJRhTgxiNzEAwL7
} 
de estudos empíricos de engenharia de software. O artigo em questão está relacionado aos processos de aplicação de experimentos na educação. Entretanto, não apresenta os conceitos pedagógicos sobre o processo de ensino de experimentação.

O artigo de [Isotani et al. 2015] apresenta conceitos envolvidos sobre o uso de ontologias na área de ES, como também possibilidades de seu uso. Os autores traçam um paralelo sobre os desafios de utilizar ontologias para representação de uma domínio especifico. Tal artigo está relacionado com a pesquisa atual referente ao uso de ontologias para representação e formalização de um domínio especifico.

A tese de [Mendonça 2015] relata metodologias existente para a criação de ontologias e também compara suas possibilidades. O foco de seu trabalho em si não está relacionado ao ensino de ES ou experimentação, como também não sendo da área da Ciência da Computação. Entretanto, tal trabalho se torna importante ao se debater sobre os tipos de metodologias existentes para a criação de ontologias, tornando-o relevante para esta pesquisa.

O trabalho de [Kuhrmann and Münch 2018] traz uma reflexão sobre os cursos de ES no âmbito de fornecerem conhecimentos e habilidades que se relacionem com a prática de mercado. Os autores relatam como os experimentos ajudam a caracterizar e estudar precisamente um problema de maneira sistemática, observar fenômenos e desenvolver e avaliar soluções. O conteúdo apresentado auxilia no processo de desenvolvimento da ontologia desta pesquisa ao observar os conteúdos abordados no processo de ensino de ES por meio de experimentação.

Os trabalhos discutidos não apresentam uma formalização sobre os conceitos correlatos ao ensino de experimentação em ES ou a sua representação por ontologias e REAs.

\section{Referências}

[Alves et al. 2012] Alves, L. M., Machado, R. J., and Ribeiro, P. (2012). Experimental software engineering in educational context. In QUATIC, pages 336-341.

[de Almeida Biolchini et al. 2007] de Almeida Biolchini, J. C., Mian, P. G., Natali, A. C. C., Conte, T. U., and Travassos, G. H. (2007). Scientific research ontology to support systematic review in software engineering. Advanced Engineering Informatics, 21(2):133-151.

[Forsyth 2018] Forsyth, D. (2018). Probability and Statistics for Computer Science. Springer, Cham.

[Furtado 2018] Furtado, V. d. R. (2018). Diretrizes para avaliação de qualidade de quasiexperimentos e experimentos controlados em linha de produto de software. Master's thesis, Prog. Pós-Grad. Ciência da Computação - Universidade Estadual de Maringá.

[Host 2002] Host, M. (2002). Introducing empirical software engineering methods in education. In $C S E E \& T$, pages $170-179$.

[Isotani et al. 2015] Isotani, S., Bittencourt, I. I., Barbosa, E. F., Dermeval, D., and Paiva, R. O. A. (2015). Ontology driven software engineering: a review of challenges and opportunities. IEEE Latin America Transactions, 13(3):863-869.

[Kuhrmann 2017] Kuhrmann, M. (2017). Teaching empirical software engineering using expert teams. In SEUH, pages 20-31. 
[Kuhrmann and Münch 2018] Kuhrmann, M. and Münch, J. (2018). Enhancing software engineering education through experimentation: an experience report. In ICE/ITMC, pages 1-9. IEEE.

[MEC 2015] MEC (2015). Diretrizes Curriculares de Cursos da Área de Computação e Informática. http://www.inf.ufrgs.br/site/wp-content/uploads/2016/ 05/diretrizes_curriculares.pdf/-Acessado em: 01 set.

[Mendonça 2015] Mendonça, F. M. (2015). OntoForInfoScience: metodologia para construção de ontologias pelos cientistas da informação-Uma aplicação prática no desenvolvimento da ontologia sobre componentes do sangue humano (HEMONTO). $\mathrm{PhD}$ thesis, Escola de Ciência da Informação - Universidade Federal de Minas Gerais.

[Mendonça and Soares 2017] Mendonça, F. M. and Soares, A. L. (2017). Construindo ontologias com a metodologia ontoforinfoscience: uma abordagem detalhada das atividades do desenvolvimento ontológico. Ciência da Informação, 46(1).

[Munezero et al. 2017] Munezero, M., Yaman, S., Fagerholm, F., Kettunen, P., Mäenpää, H., Mäkinen, S., Tiihonen, J., Riungu-Kalliosaari, L., Tuovinen, A.-P., Oivo, M., Münch, J., and Männistö, T. (2017). Continuous Experimentation Cookbook - An introduction to systematic experimentation for software-intensive businesses. DIMECC, Helsinki, Finland.

[Paiva 2011] Paiva, S. R. (2011). Uma revisão sistemática das pesquisas realizadas sobre a melhoria no ensino de engenharia de software. Technical report. Relatório Técnico-UFPB/CCEN.

[Siy and Wu 2009] Siy, H. and Wu, Y. (2009). An ontology to support empirical studies in software engineering. In 2009 International Conference on Computing, Engineering and Information, pages $12-15$. IEEE.

[Vignando et al. 2020] Vignando, H., Furtado, V. R., Teixeira, L. O., and OliveiraJr, E. (2020). OntoExper-SPL: An ontology for software product line experiments. In Int. Conf. Enterprise Information Systems, pages 401-408. SciTePress.

[Wohlin 2007] Wohlin, C. (2007). Empirical software engineering: Teaching methods and conducting studies. In Basili, V. R., Rombach, D., Schneider, K., Kitchenham, B., Pfahl, D., and Selby, R. W., editors, Empirical Software Engineering Issues: Critical Assessment and Future Directions, pages 135-142. Springer Berlin Heidelberg, Berlin, Heidelberg.

[Wohlin et al. 2012] Wohlin, C., Runeson, P., Höst, M., Ohlsson, M. C., Regnell, B., and Wesslén, A. (2012). Experimentation in software engineering. Springer Science \& Business Media. 